\title{
The energy dependence of the tetraquark production cross section
}

\author{
F. Carvalho, ${ }^{a}$ E.R. Cazaroto ${ }^{* ; b}$ V.P. Gonçalves ${ }^{c}$ and F.S. Navarra ${ }^{b}$ \\ ${ }^{a}$ Departamento de Ciências Exatas e da Terra, Universidade Federal de São Paulo, Campus \\ Diadema, Rua Prof. Artur Riedel, 275, Jd. Eldorado, 09972-270, Diadema, SP, Brazil \\ ${ }^{b}$ Instituto de Física, Universidade de São Paulo, CEP 05315-970 São Paulo, SP, Brazil \\ ${ }^{c}$ Instituto de Física e Matemática, Universidade Federal de Pelotas, CEP 96010-900, Pelotas, \\ RS, Brazil.
}

E-mail: cazaroto@if.usp.br

\begin{abstract}
We develop a model to estimate the production cross section of heavy tetraquarks as a function of the center of mass energy. The model employs two formalisms, namely the Double Parton Scattering (DPS) processes and the Color Evaporation Model (CEM). In a previous work we used our model to estimate the production cross section of the $\mathrm{X}(3872)$ and of the $\mathrm{T}_{4 c}$ in proton-proton collisions at the LHC. Now we employ it to estimate the production cross section of the $\mathrm{T}_{4 b}$, a tetraquark composed by two $b \bar{b}$ pairs, and of the $\mathrm{T}_{2 b c}$, a tetraquark composed by a $b \bar{b}$ and a $c \bar{c}$, at the energies of the LHC.
\end{abstract}

Fourth Annual Large Hadron Collider Physics

13-18 June 2016

Lund, Sweden

\footnotetext{
* Speaker.

${ }^{\dagger}$ I am thankful to the Brazilian Funding Agency FAPESP for supporting my participation in the conference.
} 


\section{Introduction}

The existence of exotic hadrons has been firmly established (see, e.g., [1] and references therein for a review) and the next step is to determine their structure. One of the most popular models used to describe them is the tetraquark, where two quark-antiquark pairs are confined in a compact region of space. However, it is not known a way to calculate the tetraquark production cross section. In Refs. [2] and [3] we give a step in this direction. We proposed a model to calculate the tetraquark production cross section as a function of the center of mass energy in protonproton collisions. In [2] we used approximate analytical expressions for the gluonic distributions $\left(g(x)=1 / x^{1+\lambda}\right.$, with $\left.\lambda \geq 0\right)$ and for the $g g \rightarrow c \bar{c}$ elementary cross section $\left(\sigma_{g_{1} g_{2} \rightarrow c \bar{c}}=\alpha_{s} / x_{1} x_{2} s\right)$ and predicted a much faster growth with the energy for the production of the $T_{4 c}$ when compared with the production of open charm. Here $T_{4 c}$ stands for a tetraquark composed by two $c \bar{c}$ pairs. In summary, we obtained: $\sigma_{4 D} \propto s^{2 \lambda}(\ln s)^{2}$ and $\sigma_{T_{4 c}} \propto s^{1+2 \lambda}(\ln s)$. In Ref. [3] we used our model with more reliable gluonic pdf's and with the correct LO pQCD expression for the gluon-gluon elementary cross section and made predictions for the production cross section of the $\mathrm{X}(3872)$ and of the $T_{4 c}$ in proton-proton collisions at the energies of the LHC. For $\sqrt{s}=14 \mathrm{TeV}$ we obtained: $\sigma_{X} \approx 45$ $\mathrm{nb}$ and $\sigma_{T_{4 c}} \approx 7 \mathrm{nb}$. In this work we use this model to calculate the production cross section of the $T_{4 b}$ and of the $T_{2 b c}$. The first is a tetraquark composed by two $b \bar{b}$ pairs while the second is a tetraquark composed by a $b \bar{b}$ and a $c \bar{c}$.

\section{A model for the production of tetraquarks}

Our model for the tetraquark production is described in two steps. The first step is the production of two quark-antiquark pairs with invariant mass $M_{12}$ and $M_{34}$ in the double parton scattering (DPS) process. This is the process where two gluons from the hadron target scatter independently with two gluons from the hadron projectile, producing a quark-antiquark pair each. The second step is the coalescence of the clusters $M_{12}$ and $M_{34}$ in a compact four quark state of mass $M$, and its subsequent transition to the final tetraquark state $M_{T}$. This process is described by employing the main ideas of the color evaporation model (CEM), which successfully describes the production of charmonium. In this approach the clusters $M_{12}$ and $M_{34}$ are kinematically bound into the compact four quark state $M$, which in turn exchanges gluons with the hadronic color field to become color neutral and to acquire the correct final state tetraquark mass $M_{T}$. The gluons exchanged in this process carry energy $\Delta$ that goes from almost zero up to the $\Lambda_{Q C D}$ scale.

Our expression for the tetraquark production cross section was derived in Ref. [3], and is given by:

$$
\begin{aligned}
\sigma_{T}=\frac{F_{T}}{\sigma_{e f f}} & {\left[\frac{1}{s} \int d y_{12} \int d M_{12}^{2} g\left(\bar{x}_{1}, \mu^{2}\right) g\left(\bar{x}_{2}, \mu^{2}\right) \sigma_{g_{1} g_{2} \rightarrow c \bar{c}}\right] } \\
\times & {\left[\frac{1}{s} \int d y_{34} \int d M_{34}^{2} g\left(\bar{x}_{3}, \mu^{2}\right) g\left(\bar{x}_{4}, \mu^{2}\right) \sigma_{g_{3} g_{4} \rightarrow b \bar{b}}\right] } \\
\times & \Theta\left(1-\bar{x}_{1}-\bar{x}_{3}\right) \Theta\left(1-\bar{x}_{2}-\bar{x}_{4}\right) \\
\times & \Theta\left(M_{12}^{2}-4 m_{c}^{2}\right) \Theta\left(M_{34}^{2}-4 m_{b}^{2}\right) \delta\left(y_{34}-y_{12}\right)
\end{aligned}
$$

where $\sigma_{\text {eff }} \simeq 15 \mathrm{mb}$ for proton-proton collisions is the effective cross section that appears in the DPS pocket formula, which is the starting formula for our model. Here $y_{12}\left(y_{34}\right)$ is the rapidity of 
the cluster with invariant mass $M_{12}\left(M_{34}\right)$. Following the scheme of the CEM we introduced the parameter $F_{T}$ which represents the probability for the four-quark system to evolve to a particular tetraquark $T$ state. $F_{T}$ is considered as energy-momentum and process independent, and after being determined by equalling above formula to the tetraquark production cross section at a given energy the model can be used to predict, with no additional free parameter, the tetraquark production cross section to any other value of the energy. All other terms of Eq. (2.1) are explicitly written in Ref. [3]. As in [3] we use the Martin-Roberts-Stirling-Thorne (MRST) gluon distribution [4] for $g\left(\bar{x}, \mu^{2}\right)$ and $\Delta \approx \Lambda_{Q C D} \approx 200 \mathrm{GeV}$ as the maximum energy carried by the gluons exchanged in the transition $M \rightarrow M_{T}$.

\section{Predictions for $T_{4 b}$ and $T_{2 b c}$ production cross sections}

The $T_{4 b}$ and the $T_{2 b c}$ are two hypothetical tetraquarks that have not yet been found experimentally, but at least in principle they may exist. Recently their possible quantum numbers were determined in Ref. [5] by using the diquark model. Unfortunately, the arbitrary number of gluons exchanged by the cluster $M$ in the transition to the final state makes our model lose any control over the quantum numbers of the tetraquark produced, except for its mass. In the case of the $T_{4 b}$ the authors of [5] found three possible states, all of them with masses very close to $M_{T_{4 b}}=18.8$ $\mathrm{GeV}$. For the $T_{2 b c}$ six different states were found, all with masses close to $M_{T_{2 b c}}=12.5 \mathrm{GeV}$. We assume these values in our calculations. Since there is no experimental data on the production of these tetraquarks, for the time being the best we can do is to make a guess of reasonable values for their production cross sections at a given energy so as to determine the constant $F_{T}$, and then make predictions for higher energies. This is what we did to make estimations for the $T_{4 c}$ in Ref. [3]. The production cross section of the X(3872) was measured by the CMS collaboration [6] at 7 $\mathrm{TeV}$, so we tried to guess a value for $\sigma_{T_{4 c}}$ at this energy based on the value measured for $\sigma_{X}$. It happens that energetically speaking the $T_{4 c}$ is more difficult to be produced than the $\mathrm{X}(3872)$ because this last is composed by a $c \bar{c}$ and a light $q \bar{q}$. Based on this observation we took the experimental value of $\sigma_{X}(\approx 30 \mathrm{nb})$ and multiplied by a penalty factor given by $\sigma_{c \bar{c}} \sigma_{c \bar{c}} / \sigma_{c \bar{c}} \sigma_{q \bar{q}} \simeq 0.12$, obtaining $\sigma_{T_{4 c}}=0.12 \times \sigma_{X}=3.6 \mathrm{nb}$. Following this same strategy let us guess values for $\sigma_{T_{4 b}}$ and for $\sigma_{T_{2 b c}}$ at $7 \mathrm{TeV}$ based on the value determined for $\sigma_{T_{4 c}}$ in Ref. [3]. We have:

$$
\sigma_{T_{4 b}}=\frac{\sigma_{b \bar{b}} \cdot \sigma_{b \bar{b}}}{\sigma_{c \bar{c}} \cdot \sigma_{c \bar{c}}} \sigma_{T_{4 c}} \simeq 4.2 \times 10^{-3} \mathrm{nb} \quad ; \quad \sigma_{T_{2 b c}}=\frac{\sigma_{b \bar{b}} \cdot \sigma_{c \bar{c}}}{\sigma_{c \bar{c}} \cdot \sigma_{c \bar{c}}} \sigma_{T_{4 c}} \simeq 0.12 \mathrm{nb}
$$

where the experimental values $\sigma_{c \bar{c}} \simeq 8.5 \mathrm{mb}$ [7] and $\sigma_{b \bar{b}} \simeq 288 \mu \mathrm{b}$ [8] at $7 \mathrm{TeV}$ were used. Now we use the values determined in (3.1) to fix the constants $F_{T_{4 b}}$ and $F_{T_{2 b c}}$, respectively to the tetraquarks $T_{4 b}$ and $T_{2 b c}$. Then we plot their production cross sections as functions of the energy in Fig. 1. On the left we have the curves for the $T_{4 b}$ and on the right we have the ones for the $T_{2 b c}$. It is difficult to determine the uncertainty of this calculation. However we verified that our results are very sensitive to the masses chosen for the heavy quarks. So we varied the charm mass from 1.2 to $1.5 \mathrm{GeV}$ and the bottom mass from 4.3 to $4.6 \mathrm{GeV}$ and determined an error band based on these choices. In each plot the upper curve corresponds to the lightest masses for the heavy quarks, while the lower curve corresponds to the heavier masses. The points at 7 and $14 \mathrm{TeV}$ were chosen so that the average value of the curves cross them. 

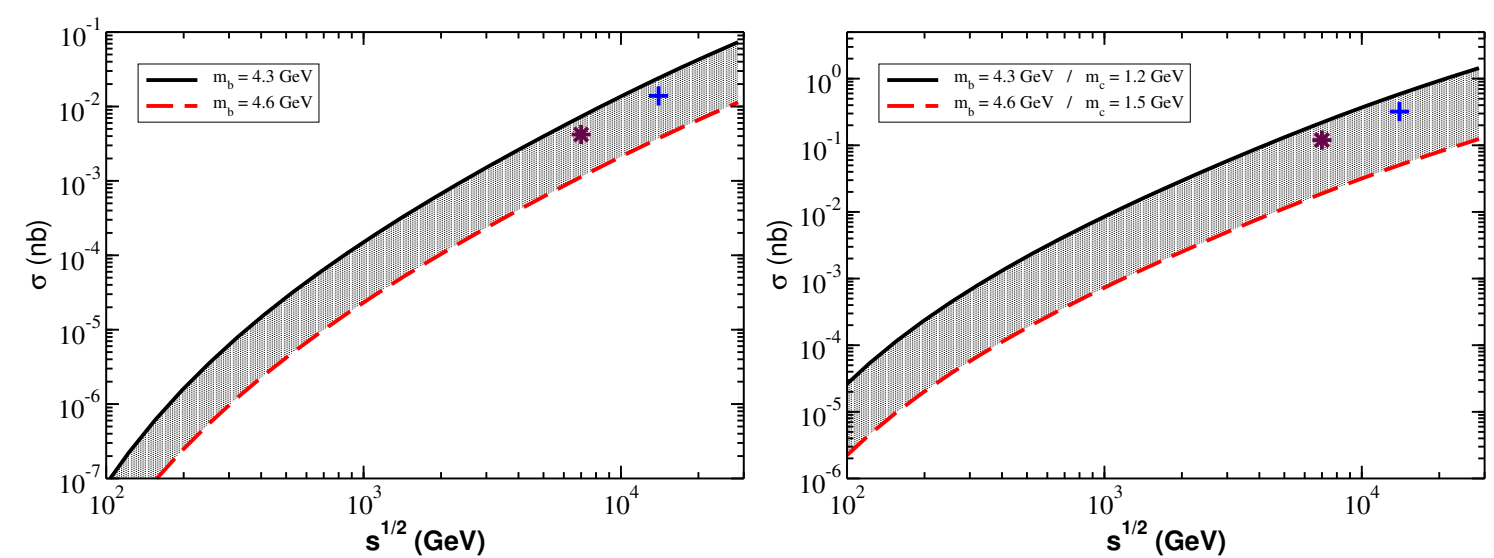

Figure 1: Prediction for the tetraquark production cross section as a function of the center of mass energy in proton-proton collisions. Left: prediction for the $T_{4 b}$; and Right: prediction for the $T_{2 b c}$. The asterisk is the point used to fix the constant $F_{T}$ at $\sqrt{s}=7 \mathrm{TeV}$ and the cross is the prediction of the model for $\sqrt{s}=14$ $\mathrm{TeV}$.

For $14 \mathrm{TeV}$ our model predicts:

$$
\sigma_{T_{4 b}} \approx(13.9 \pm 10.1) \times 10^{-3} n b \quad ; \quad \sigma_{T_{2 b c}} \approx(0.32 \pm 0.27) n b
$$

The large band error shows the sensitiveness of the model to the choice of the heavy quark masses.

\section{Acknowledgments}

This work was partially financed by the Brazilian funding agencies CAPES, CNPq, FAPESP and FAPERGS.

\section{References}

[1] A. Esposito, A. L. Guerrieri, F. Piccinini, A. Pilloni, and A. D. Polosa, Int. J. Mod. Phys. A 30, 1530002 (2015); N. Brambilla et al., Eur. Phys. J. C 71, 1534 (2011).

[2] F. Carvalho, E.R. Cazaroto, V.P. Gonçalves and F.S. Navarra, J. Phys. Conf. Ser. 706 (2016) no.4, 042003.

[3] F. Carvalho, E.R. Cazaroto, V.P. Gonçalves and F.S. Navarra, Phys. Rev. D 93, 034004 (2016).

[4] A.D. Martin, R.G. Roberts, W.J. Stirling, and R.S. Thorne, Eur. Phys. J. C 4, 463 (1998).

[5] A.V. Berezhnoy, A.V. Luchinsky, and A. A. Novoselov, Phys. Rev. D 86, 034004 (2012); A.V. Berezhnoy, A. K. Likhoded, and A. A. Novoselov, Phys. Rev. D 87, 054023 (2013)

[6] S. Chatrchyan et al. (CMS Collaboration), J. High Energy Phys. 04 (2013) 154.

[7] B. Abelev et al. (ALICE Collaboration), J. High Energy Phys. 07 (2012) 191.

[8] B. Abelev et al. (ALICE Collaboration), J. High Energy Phys. 11 (2012) 065. 